\title{
Protective role of STVNa in myocardial ischemia reperfusion injury by inhibiting mitochondrial fission
}

\author{
Xiaoou Sun ${ }^{1}$, Yingying Yang ${ }^{2}$, Yanxiang $\mathrm{Xie}^{2}$, Xingjuan Shi ${ }^{3}$, Lijie Huang ${ }^{2}$ and Wen \\ $\operatorname{Tan}^{1}$ \\ ${ }^{1}$ Institute of Biomedical and Pharmaceutical Sciences, Guangdong University of Technology, Guangzhou 510006, China \\ ${ }^{2}$ School of Bioscience and Bioengineering, South China University of Technology, Guangzhou 510006, China \\ ${ }^{3}$ Key Laboratory of Developmental Genes and Human Disease, Institute of Life Sciences, Southeast University, Nanjing \\ 210096, China
}

Correspondence to: Wen Tan, email: went@gdut.edu.cn

Keywords: STVNa; mitochondrial; ischemia reperfusion injury; cardioprotective

Received: May 30, 2017 Accepted: November 14, $2017 \quad$ Published: December 05, 2017

Copyright: Sun et al. This is an open-access article distributed under the terms of the Creative Commons Attribution License 3.0 (CC BY 3.0), which permits unrestricted use, distribution, and reproduction in any medium, provided the original author and source are credited.

\section{ABSTRACT}

It has been reported that isosteviol, a widely known sweeteners, can protect against myocardial ischemia-reperfusion (IR) injury in isolated guinea pig heart. Here, we aim to confirm the cardioprotective effect of its sodium salt, isosteviol sodium (STVNa), against IR injury and its potential molecular mechanism in H9c2 cardiomyocytes. STVNa significantly improved cell viability, restored mitochondrial membrane potential, decreased cellular reactive oxygen species generation, and inhibited cell apoptosis. Furthermore, STVNa treatment changed the morphology of mitochondria from fragmented, discontinuous forms to normal elongated, tubular forms. Cyto-immunofluorescence and western blot analysis revealed that STVNa inhibited mitochondrial fission proteins dynamin-related protein 1 (Drp1), and mitochondrial fission 1 (Fis1), thus plays a key role in cardioprotection. These findings, for the first time, suggest that STVNa can protect against myocardial IR injury through reverse mitochondrial fission.

\section{INTRODUCTION}

Isosteviol, is the acid hydrolysate product of stevioside, a widely known sweeteners. In recent years, a number of studies have investigated the anti-hyperglycemic [1], anti-hypertensive [2], antiinflammatory [3] and anti-tumor effects of isosteviol [4]. The cardioprotective effect of isosteviol in ischemiareperfusion (IR) injury in isolated guinea pig heart has also been reported [5, 6]. Although potassium channels and calcium channels have been reported to be involved in the action of isosteviol, the potential cardioprotective mechanism of isosteviol is not yet clear [7].

Myocardial IR injury is a major cause of death and disability worldwide. The pathophysiology of myocardial reperfusion injury can induce arrhythmias, myocardial stunning, oxidative stress, intracellular $\mathrm{Ca}^{2+}$ overload, and mitochondrial permeability transition pore (mPTP) opening [8]. Processes involved in myocardial cell IRinjury include inflammation, excitotoxicity, mitochondrial dysfunction, and oxidative stress, and cell apoptosis $[8$, 9]. Mitochondria are important regulators of cell growth, and are highly dynamic organelles involved in fusion and fission in response to ischemia or other types of oxidative stress [9]. The morphology of normal mitochondria is tubular, elongated and interconnected. However, when undergoing IR-injury the mitochondrial permeability transition pore opens, mitochondria dynamic balance would be lost, lead to changes in morphology and the mitochondria are fragmented and discontinuous. It has been shown that dynamin-related protein 1 (Drp1), and mitochondrial fission 1 (Fis1) play key roles in this process [10]. Changes in mitochondria morphology also have an impact on mitochondrial membrane potential $\left(\psi_{\mathrm{m}}\right)$, impair the redox buffer system and induce reactive oxygen species (ROS) production [11]. Mitochondrial fission proteins affect the function of the organelle and eventually integrated cellular signaling cascades, including apoptosis. In the present study, we found that isosteviol sodium (STVNa), the sodium salt of isosteviol protects 
H9c2 cardiomyocytes against IR injury through inhibition of the mitochondrial fission pathway.

\section{RESULTS}

\section{Effect of STVNa on H9c2 cell viability following different ischemia times}

H9c2 cells were subjected to $0,30,60,90,120$, and $150 \mathrm{~min}$ ischemia followed by reperfusion with or without STVNa for $90 \mathrm{~min}$. The results showed that with extended ischemia time, cell viability gradually decreased to $30.4 \%$ $\pm 1.3 \%$ compared to the control. At that time IR injury was serious and unable to recover. However, following ischemia for 30, 60, and $90 \mathrm{~min}, 10 \mu \mathrm{M}$ STVNa showed a significant protective effect $(P<0.05$, Figure 1B). Therefore, we chose an ischemia time of $90 \mathrm{~min}$ for the following experiment as cell viability was $69.6 \% \pm 1.3 \%$.

\section{STVNa restored mitochondrial membrane potential $(\Delta \psi)$ during IR}

Decreased cell viability is generally associated with a disturbance in mitochondrial function. The dissipation of $\Delta \psi$ is an indication of failing mitochondria. We assessed the effect of STVNa on $\Delta \psi$ using the membrane sensitive dye JC-1. As shown in Figure 2, $90 \mathrm{~min}$ of ischemia followed by $90 \mathrm{~min}$ of reperfusion resulted in a marked decrease in $\Delta \psi(\mathrm{R} / \mathrm{G}$ ratio: $0.328 \pm 0.006$ vs $0.944 \pm 0.03$ in the control group). Cells treated with 1,10 , and 100 $\mu \mathrm{M}$ STVNa partially recovered $\Delta \psi$ in a dose-dependent manner $(P<0.05)$. Diazoxide was used as a positive control $(P<0.05)$. Furthermore, 10 and $100 \mu \mathrm{M}$ STVNa had a better effect than $100 \mu \mathrm{M}$ diazoxide in maintaining $\Delta \psi(P<0.05)$.

\section{STVNa decreased IR-induced intracellular ROS production}

Oxidative stress is the primary contributor in IR injury. It can induce modifications in mitochondrial proteins, DNA and lipids, which inhibit energy production and contractile function, eventually leading to cell apoptosis [12]. To determine whether STVNa inhibited oxidative stress, intracellular ROS production was measured by DCFH-DA staining. Figure 3 shows that IR induced a burst in ROS production (mean fluorescence intensity: $1.688 \pm 0.024$ vs $1.030 \pm 0.013$ in the control). STVNa $(1,10$ and $100 \mu \mathrm{M})$ significantly reduced ROS accumulation $(P<0.05)$.

\section{STVNa inhibited IR-induced cell apoptosis}

To determine the protective effect of STVNa on cell apoptosis during H9c2 cell IR injury. Cells from different treatment groups were evaluated by the DNA-binding fluorescent dye DAPI and TUNEL. The morphology of a normal cell nucleus is large and uniform, however, condensation and enhanced fluorescence intensity were seen in apoptotic cells (Figure 4A). Quantitation of the extent of NCI using Image-Pro Plus analysis revealed that the proportion of $\mathrm{NCI}$ in the IR group was $50.22 \% \pm 4.6 \%$ and decreased to $23.64 \% \pm 3.2 \%(P<0.05), 18.52 \% \pm$ $3.4 \%(P<0.05)$, and $15.65 \% \pm 1.8 \%(P<0.05)$ following treatment with 1,10 , and $100 \mu \mathrm{M}$ STVNa, respectively. TUNEL method was also used to examine the occurrence of cell apoptosis (Figure 4C, 4D). The percent of TUNEL positive cells was markedly increased in IR group compared to control, while the number of apoptosis cells in $10 \mu \mathrm{M}$ STVNa treatment group was obviously reduced $(P<0.05)$. We further examined caspase-3 activation of cells with different treatment, and found that STVNa reduces the effect of IR-injury on caspase-3 activation (Figure 4E).

\section{Effect of STVNa on mitochondrial morphology in confocal images}

We examined the changes in mitochondrial morphology during IR injury. As shown in Figure 5, the morphology of mitochondria became fragmented or discontinuous after IR. However, this effect was largely inhibited by $10 \mu \mathrm{M}$ STVNa. Taken together, pharmacological treatment with STVNa inhibited abnormal mitochondrial fission and reduced cell death.

\section{STVNa inhibits the expression of proteins related to mitochondrial morphology}

To determine the underlying molecular mechanism of STVNa in the inhibition of mitochondrial fragmentation in myocardial IR injury, we assessed the expression of the mitochondrial fission related proteins, Drp1 and Fis1, by immunofluorescence and western blot analysis. As shown in Figure 6, compared to the control cells, the fluorescence intensity of Drp1 and Fis1 in IR cells was increased $(P<0.05)$. Western blot analysis also revealed that the expression of Drp1 and Fis1 proteins was upregulated $(P<0.05)$. However, when treated with STVNa during reperfusion, the fluorescence intensity and protein expression of Drp1 and Fis1 were significantly decreased $(P<0.05)$. Thus, we suggest that STVNa inhibits IRinduced cardiomyocyte apoptosis through regulating the expression of mitochondrial fission proteins, which play key roles in myocardial IR injury.

\section{DISCUSSION}

Mitochondria, are the energy source in cells, and are highly dynamic organelles. They can change in number and morphology within a cell during development in response to various stimulants [13]. In myocardial IR 
injury, mitochondrial damage including $\mathrm{Ca}^{2+}$ overload, ROS overproduction, and the opening of MPTP plays a key role in cardiomyocyte apoptosis [14]. There are a number of proteins which participate in mitochondrial fusion and fission, of which Drp1 is the mostly frequently studied [15]. Fis1 is thought to act as a mitochondrial receptor for Drp1 [16]. In response to various stimulants, Drp1 translocates from the cytosol to the mitochondrion and wraps around the mitochondrion to induce fission by binding to its mitochondrial adaptors such as Fis1 [17]. It has been reported that the inhibition of mitochondrial fission can protect the heart against myocardial IR injury both in vivo and in vitro $[18,19]$. This may be a new target for IR therapy [20]. In the present study, the expression of Drp1 and Fis1 in the IR group was significantly increased accompanied by cell death. Conversely, pharmacological inhibition with STVNa improved mitochondrial morphology and reduced cell death. These changes at the molecular level also corresponded to the dynamic transformation of mitochondria morphology.

Previous studies have revealed that inhibition of Drp1 increases cell survival, decreases IR-associated calcium and ROS, and preserves mitochondrial oxygen consumption and morphology [18]. Impairment of mitochondrial integrity results in ROS production, DNA damage and activation of cell death [21]. In the current study, the rat ventricular H9c2 cell line was used. This cell line has been used extensively in studies investigating signal transduction mechanisms in cardiomyocytes as it retains properties of the signaling pathways of cardiomyocytes. It is also suitable for in vivo models to simulate IR injury due to its high sensitivity [22]. In our experiment, when cell viability was approximately $50 \%$ these cells were used as an IR model. STVNa significantly improved cell survival rate which was determined using the MTT assay. In subsequent experiments, the myocardial cells were subjected to 90 min of ischemia followed by

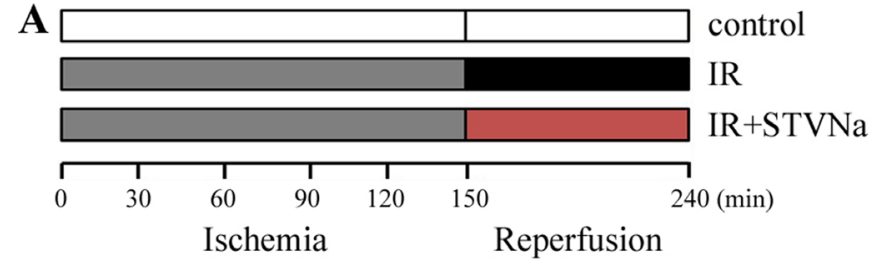

90 min of reperfusion. In ischemic heart disease and other cardiomyopathies, mitochondrial dysfunction is a key factor in inducing cell death. The inhibition of mPTP opening and mitochondrial antioxidants are two main therapeutic targets [23]. mPTP opening results in mitochondrial swelling and the depolarization of $\Delta \psi$ [24]. Oxidative stress causes cell damage due to modifications in mitochondrial proteins, DNA, and lipids [25]. It has been proved that STVNa can both preserve $\Delta \psi$ and significantly decrease reperfusion-induced ROS overproduction in a dose-dependent manner. Furthermore, compared to the selective mitochondrial ATP-sensitive potassium channel opener diazoxide, which has been reported to protect cardiac mitochondria [26, 27], STVNa has a better protective effect.

The final event in IR is cell apoptosis. Apoptosis, which is programmed cell death, is characterized by cell shrinkage and pyknosis. It is the result of chromatin condensation [28]. The cell nucleus is shrunken and irregular during apoptosis which can be detected by nucleic acid staining with DAPI due to its high affinity for DNA. The present study shows that treatment with STVNa inhibits cell apoptosis by adjusting a series of upstream signal pathways.

\section{MATERIALS AND METHODS}

\section{Chemicals and antibodies}

STVNA was provided by the Chemical Development Laboratories of Key Biological Pharmaceutical Company (Dongguan, China). The JC-1 and 2',7'-dichlorofluorescin diacetate (DCFH-DA) were purchased from Life Technology. Diazoxide, Terminal Deoxynucleotidyl Transferase-mediated dUTP Nick End

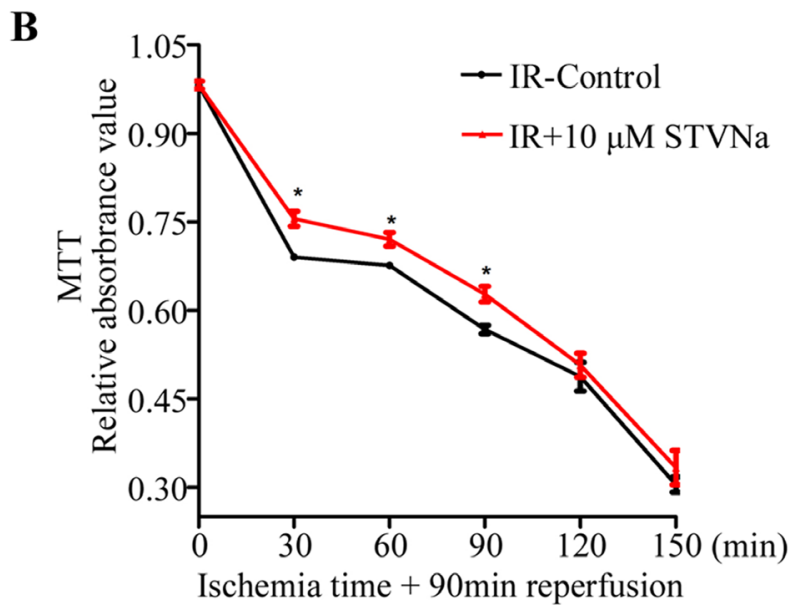

Figure 1: Effect of IR on H9c2 cell viability and the protective effect of STVNa in different ischemia conditions. (A) The protocol used to investigate the appropriate ischemia time and the effect of STVNa on cell viability in different ischemia conditions. (B) H9c2 cells were subjected to $0,30,60,90,120$, and $150 \mathrm{~min}$ ischemia followed by reperfusion with or without $10 \mu \mathrm{M}$ STVNa for 90 min. Cell viability was assessed in the different treatment groups by the MTT assay. Data are shown as mean \pm S.E.M in four independent experiments. ${ }^{*} P<0.05$ vs. IR. 
Labelling (TUNEL) and 3-(4, 5-dimethylthiazolyl-2)-2, 5-diphenyl-2-H-tetrazolium bromide (MTT) were from Sigma-Aldrich. 4',6-diamidino-2-phenylindole (DAPI), and antibodies against Drp1, Fis1, Caspase-3, Cleaved Caspase-3 and b-actin were purchased from Abcam. Horseradish peroxidase-conjugated secondary antibodies were purchased from Santa Cruz Biotechnology, and fluorescein-and rhodamine-conjugated secondary antibodies were from Jackson ImmunoResearch Laboratories.

\section{Cell culture and IR injury model}

The H9c2 cells were obtained from the Type Culture Collection of the Chinese Academy of Sciences (Shanghai, China) and cultured according to the manuals. IR injury was induced as previously reported. Briefly, H9c2 cells were seeded in a six-well plate containing $2 \times 10^{5}$ cells/ well. After adherence for $24 \mathrm{~h}$, the medium was replaced with ischemia buffer $(137 \mathrm{mM} \mathrm{NaCl}, 15.8 \mathrm{mM} \mathrm{KCl}$, $0.49 \mathrm{mM} \mathrm{MgCl}, 0.9 \mathrm{mM} \mathrm{CaCl}_{2}, 4 \mathrm{mM}$ HEPES, $10 \mathrm{mM}$
A control
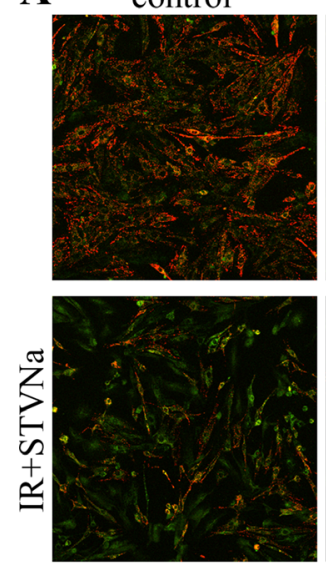

$1 \mu \mathrm{M}$
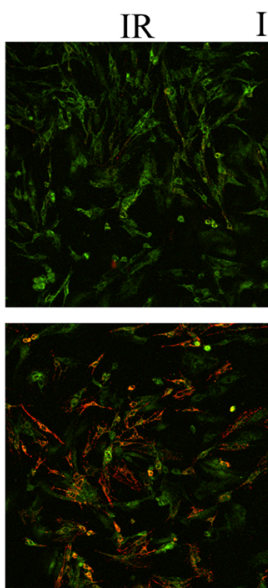

$10 \mu \mathrm{M}$
IR $+100 \mu \mathrm{M}$ diazoxide
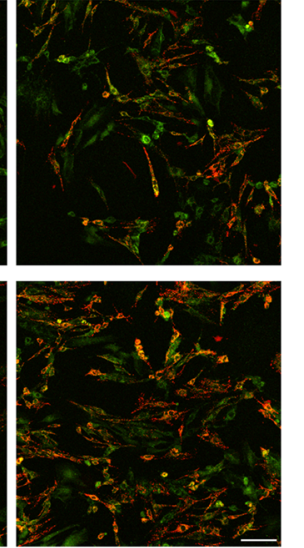

$100 \mu \mathrm{M}$
B

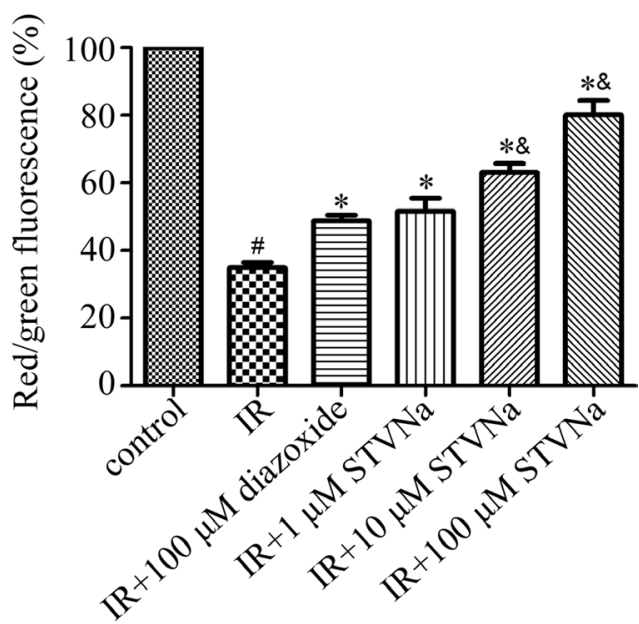

Figure 2: Effect of STVNa on mitochondrial membrane potential after IR. (A) Confocal images of mitochondrial potential following JC-1 staining. Scalebar: $100 \mu \mathrm{m}$. (B) Graph of red-to-green $(\mathrm{R} / \mathrm{G})$ fluorescence intensity. Diazixide was used as a positive control. Images representative of three individual experiments. Data are expressed as percentages of the control level. All values are expressed as means \pm S.E.M. ${ }^{\#} P<0.05$ vs. control; ${ }^{*} P<0.05$ vs. IR; ${ }^{\&} P<0.05$ vs. $100 \mu \mathrm{M}$ diazoxide.

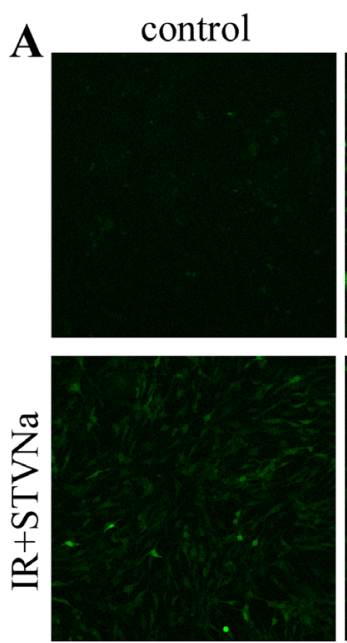

$1 \mu \mathrm{M}$
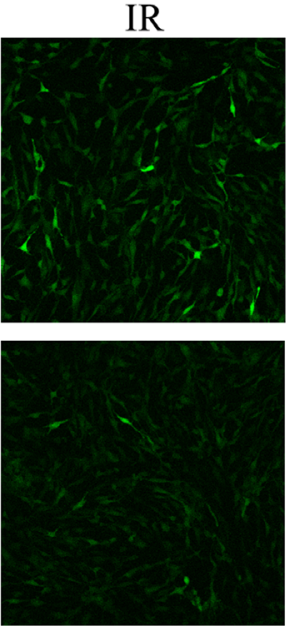

$10 \mu \mathrm{M}$

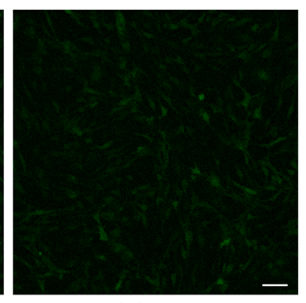

$100 \mu \mathrm{M}$
B

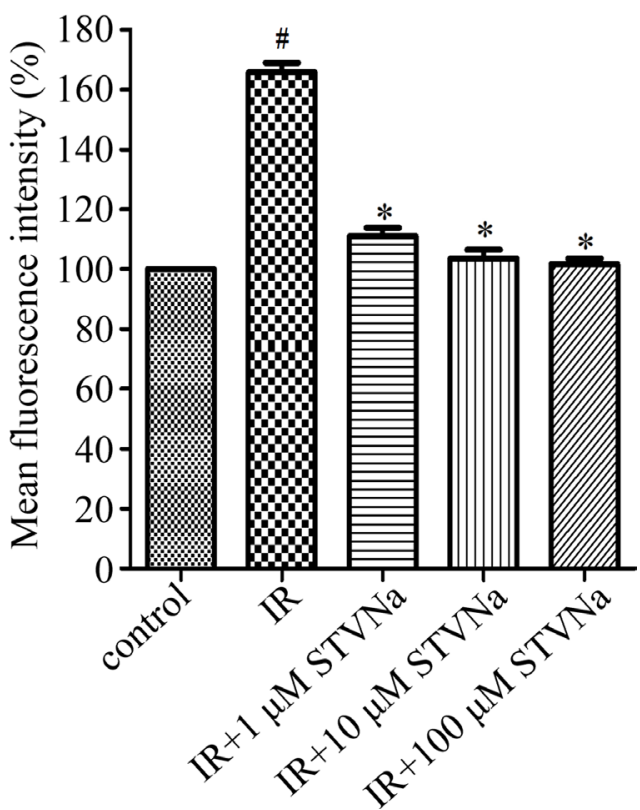

Figure 3: STVNa decreased IR-induced intracellular ROS production. (A) Confocal images of ROS. Intracellular ROS production was measured by DCFH-DA staining. Scalebar: $100 \mu \mathrm{m}$. (B) Graph of ROS levels. Images representative of three individual experiments. Data are expressed as percentages of the control level. All values are expressed as means \pm S.E.M. ${ }^{\#} P<0.05$ vs. control; ${ }^{*} P$ $<0.05$ vs. IR. 
A

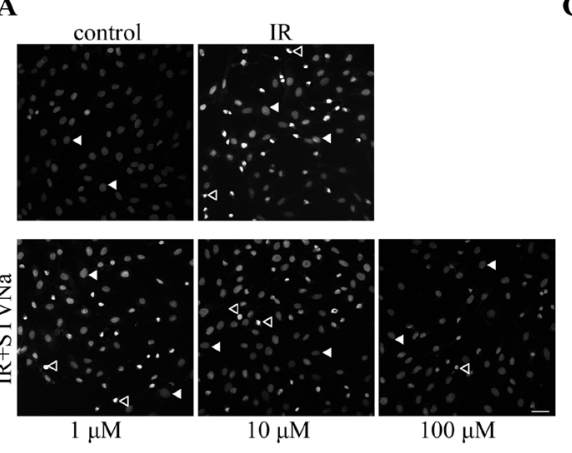

C

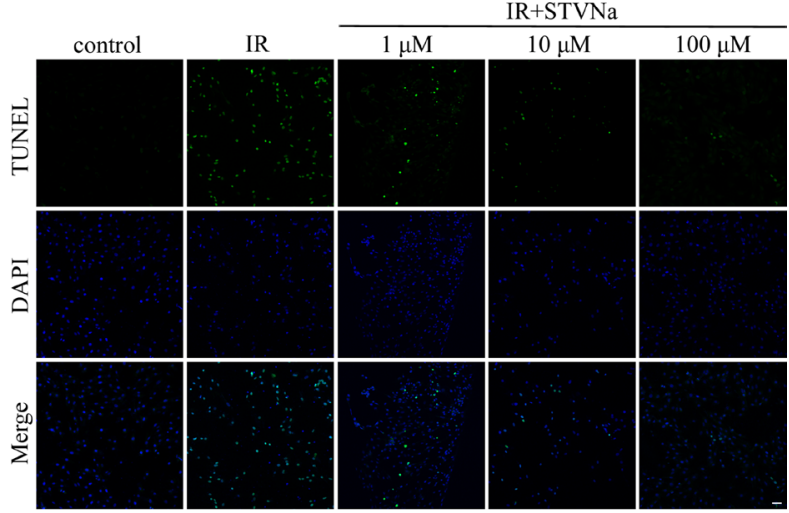

$\mathbf{E}$
B

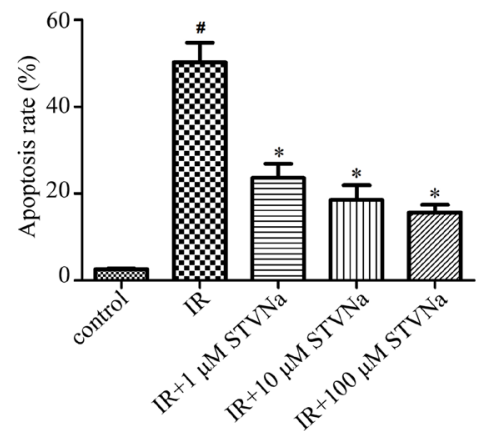

D

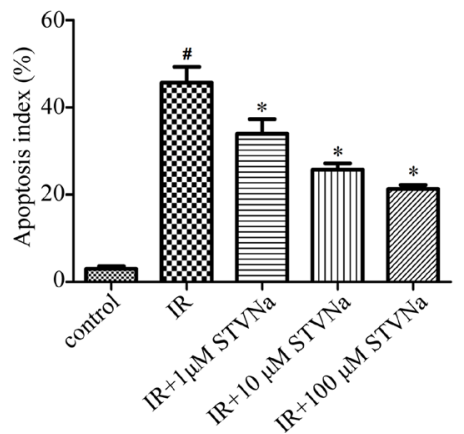

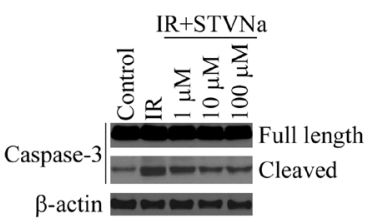

$\beta$-actin $\mathrm{B}=\mathrm{m} \rightarrow \mathrm{m}$

Figure 4: STVNa inhibited IR-induced cell apoptosis. (A) Confocal images of apoptosis associated with nuclear condensation in $\mathrm{H} 9 \mathrm{c} 2$ cardiomyocytes. H9c2 cells from different treatment groups were evaluated by the DNA-binding fluorescent dye DAPI and images were then taken under the fluorescence microscope. The arrowhead and open arrowhead indicate respectively. Scalebar: $100 \mu \mathrm{m}$. $(\mathbf{B})$ Experiments were performed as in (A), and the ratio of NCI cells in each group was counted. $n=100$ per group. (C) The fluorescence images of TUNEL staining. H9c2 cells from different treatment groups were evaluated by TUNEL (green) and DNA dye DAPI (blue) and images were then taken under the fluorescence microscope. (D) Experiments were performed as in (C), and the percent of apoptosis cells were decided by TUNEL positive cells (green) to the total DAPI staining cells (blue). The data were then normalized to the control group. All values are expressed as means \pm S.E.M, $n=100$ per group. ${ }^{\#} P<0.05$ vs. control; ${ }^{*} P<0.05$ vs. IR. (E) The activation of caspase-3 in different treatment groups was analyzed by immunoblot analysis.

A

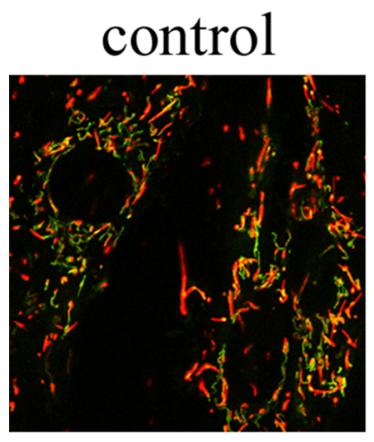

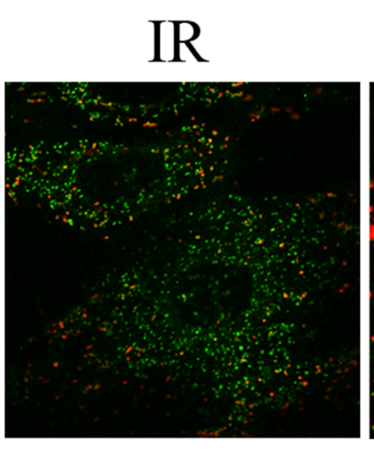

B

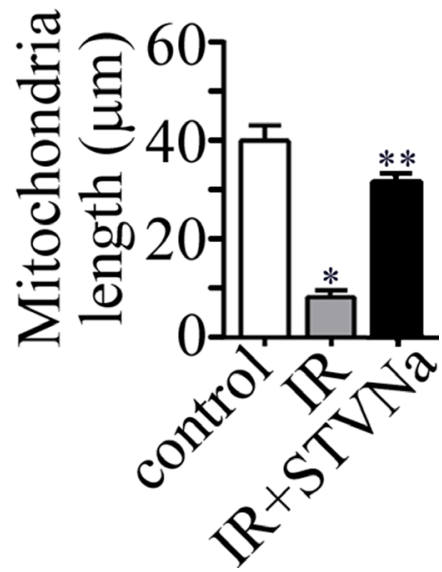

Figure 5: Changes in mitochondrial morphology during IR. (A) JC-1 staining shows the tubular mitochondrial morphology in H9c2 cells under normal conditions. Small and fragmented mitochondria were evident in cells undergoing IR. STVNa (10 $\mu$ M) recovered mitochondrial morphology to some extent. Scalebar: $100 \mu \mathrm{m}$. (B) Experiments were performed as in (A), and the length of mitochondria were measured. The data were then normalized to the control group. All values are expressed as means \pm S.E.M, $n=40$ per group ${ }^{*} P<$ $0.05 ;{ }^{* *} P<0.01$. 
2-deoxyglucose, $20 \mathrm{mM}$ sodium lactate and $1 \mathrm{mM}$ sodium dithionite, adjusted to $\mathrm{pH} 6.5$ ) to mimic ischemia in vitro. The cells were then incubated in a hypoxia chamber containing $94 \% \mathrm{~N}_{2}, 1 \% \mathrm{O}_{2}$, and $5 \% \mathrm{CO}_{2}$ for a period of time. At the end of ischemia, the buffer was discarded and replaced with fresh DMEM buffer. The cells were incubated for a further $90 \mathrm{~min}$ in a normal environment. In the drug treatment groups, 1, 10, and $100 \mu \mathrm{M}$ STVNa were added to the reperfusion buffer at the onset of reperfusion (Figure 1A).

\section{Cell viability assay}

H9c2 cells were seeded at a density of $1 \times 10^{4} /$ well in a 96-well plate and the cell IR injury model was established as described above. After adherence for $24 \mathrm{~h}$, the cells were incubated in a ischemia buffer for $90 \mathrm{~min}$. At the end of ischemia, the cells were incubated for a further $90 \mathrm{~min}$ in a normal environment. H9c2 cells were subjected to $0,30,60,90,120$, and $150 \mathrm{~min}$ ischemia followed by reperfusion with or without STVNa for 90 min. Cell viability was assessed in the different treatment groups by the MTT assay then. The MTT stock solution (5 $\mathrm{mg} / \mathrm{mL}$ ) was diluted with DMEM to a final concentration of $0.5 \mathrm{mg} / \mathrm{mL}$ and $100 \mu \mathrm{L}$ was added to each well. Following incubation for $4 \mathrm{~h}$ at $37^{\circ} \mathrm{C}$, the supernatants
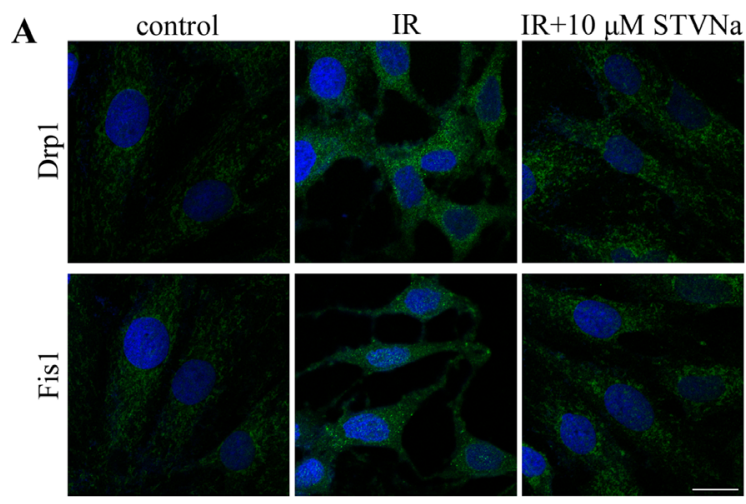

C

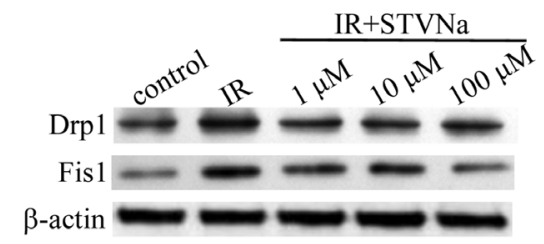

were discarded and $150 \mu \mathrm{L} /$ well DMSO was used to dissolve the formazan crystals. The optical density was measured at $490 \mathrm{~nm}$ using a microplate reader.

\section{Measurement of mitochondrial membrane potential $(\Delta \psi)$}

$\mathrm{JC}-1$ is a cationic dye that exhibits potential-dependent accumulation in mitochondria, indicated by a fluorescence emission shift from green $(525 \mathrm{~nm})$ to red $(590 \mathrm{~nm})$. Consequently, mitochondrial depolarization is indicated by a decrease in the red/green fluorescence intensity ratio. The potential sensitive color shift is due to the concentrationdependent formation of red fluorescent J-aggregates. $\mathrm{H} 9 \mathrm{c} 2$ cells in different treatments were exposed to JC-1 staining solution $(10 \mu \mathrm{g} / \mathrm{mL})$ for $20 \mathrm{~min}$ at $37^{\circ} \mathrm{C}$. The cells were then washed twice with phosphate-buffered saline (PBS) and incubated in DMEM for observation using a confocal microscope (Carl Zeiss). The ratio of red to green fluorescence intensity was analyzed as $\Delta \psi$.

\section{Assessment of intracellular ROS}

Intracellular ROS were assessed by DCFH-DA, a cell-permeable non-fluorescent probe which is de-
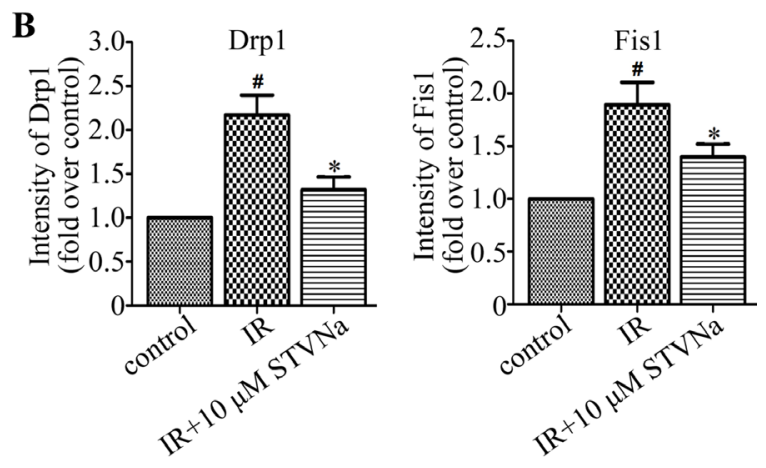

$\mathbf{D}$
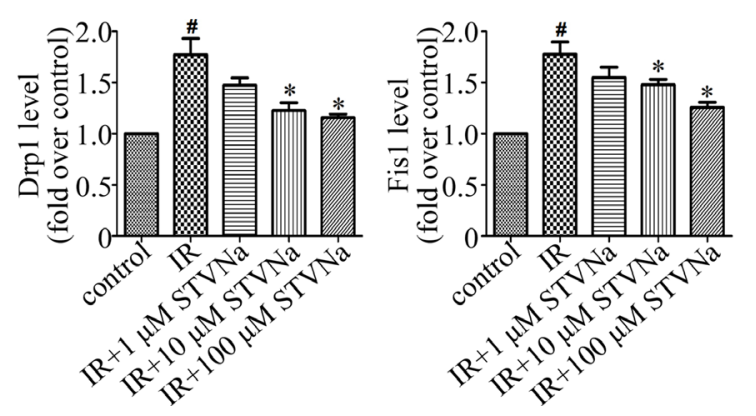

Figure 6: STVNa inhibited mitochondrial fission proteins Drp1 and Fis1. (A) H9c2 cells in each group were stained with antibodies against Drp1 and Fis1 (green) and the DNA dye DAPI (blue), and images were then taken under the fluorescence microscope. Scalebar: $50 \mu \mathrm{m}$. (B) Experiments were performed as in (A), and the levels of Drp1 and Fis1 were analyzed by measuring the fluorescence intensity in each group. The data were then normalized to the control group. (C) The levels of Drp1, Fis1 and actin were examined by immunoblotting with antibodies against Drp1, Fis1 and b-actin. (D) Experiments were performed as in (C), and the level of Drp1 and Fis1 was analyzed. Protein levels are relative to the control group. All values are expressed as means \pm S.E.M in four independent experiments. ${ }^{\#} P<0.05$ vs. control; ${ }^{*} P<0.05$ vs. IR. 
esterified intracellularly and turns to highly fluorescent 2 ',7'-dichlorofluorescein upon oxidation. At the end of reperfusion, H9c2 cells were loaded with DCFH-DA (5 $\mu \mathrm{M})$ in DMEM for $30 \mathrm{~min}$ at $37^{\circ} \mathrm{C}$. The cells were then washed three times with PBS. The DCF fluorescence intensity was measured using confocal microscopy at 488 excitation and $520 \mathrm{~nm}$ emission.

\section{Detection of apoptosis by DAPI staining}

Apoptotic cells were evaluated using the DNAbinding fluorescent dye DAPI. The percentage of nuclear condensation (NCI) in cells represents the extent of IR injury. H9c2 cells were fixed with 4\% paraformaldehyde for $20 \mathrm{~min}$ at room temperature and then incubated with DAPI $(10 \mu \mathrm{M})$ dissolved in PBS for $7 \mathrm{~min}$. After washing several times with PBS, fluorescence was visualized by confocal microscopy. The ratio of NCI cells in each group was counted using Image-Pro software.

\section{TUNEL assay}

Cells from different treatment groups were fixed and immersed in 1\% Triton X-100 for $5 \mathrm{~min}$. After washing several times with PBS, cells were incubated in a total TUNEL reaction buffer for $1 \mathrm{~h}$ at $37^{\circ} \mathrm{C}$. Then a Streptavidin-Fluorescein Labeling buffer was replaced for another $30 \mathrm{~min}$ in the same condition. Finally, the nuclei was counterstained with DAPI. The percent of apoptosis cells were decided by TUNEL positive cells (green fluorescence nuclei) to the total DAPI staining cells (blue fluorescence nuclei). Images were captured by fluorescence microscope and six random fields were calculated for apoptosis index.

\section{Determination of mitochondrial morphology}

H9c 2 cells were seeded at $3 \times 10^{4} /$ well in a $35 \mathrm{~mm}$ glass bottom culture dish. Following different treatments, the cells were loaded with JC-1 as previously described. Mitochondrial morphology was then determined using a confocal microscope equipped with a $100 \times$ oil immersion objective.

\section{Immunoblotting}

Proteins were separated by sodium dodecyl sulfatepolyacrylamide gel electrophoresis and trans-ferred onto polyvinylidene difluoride membranes (Millipore). The membranes were blocked with Tris-buffered saline containing $0.2 \%$ Tween 20 and $5 \%$ fat-free dry milk. The membranes were then incubated sequentially with primary antibodies and then horseradish peroxidase-conjugated secondary antibodies. Specific proteins were detected with the enhanced chemiluminescence detection reagent (Pierce), according to the manufacturer's protocol.

\section{Immunofluorescence microscopy}

Cells cultured on chamber slides were fixed with $4 \%$ paraformaldehyde for $30 \mathrm{~min}$, permeated in $0.5 \%$ Triton $\mathrm{X}-100 / \mathrm{PBS}$ for $20 \mathrm{~min}$ and blocked in $2 \%$ bovine serine albumin in PBS for $30 \mathrm{~min}$ at room temperature. The cells were then incubated in succession with primary antibodies and fluorescein- or rhodamine-conjugated secondary antibodies followed by staining with DAPI for $5 \mathrm{~min}$. Coverslips were mounted with $90 \%$ glycerol in PBS and examined with a confocal microscope.

\section{Statistical analysis}

Results are shown as mean \pm standard error of the mean. Statistical analysis among different groups was assessed by one-way analysis of variance with Tukey's post hoc test. A $P$ value $<0.05$ was defined as statistically significant.

\section{CONCLUSIONS}

In summary, our results indicate that STVNa inhibits IR-induced H9c2 myocardial cell apoptosis by downregulating the mitochondria fission proteins, Drp1 and Fis 1, recovering $\Delta \psi$ and decreasing intracellular ROS overproduction. STVNa may be a promising drug for the treatment of cardiovascular diseases in the future.

\section{CONFLICTS OF INTEREST}

The authors have declared that no competing financial interests exist.

\section{GRANT SUPPORT}

This work was funded in part by the National Natural Science Foundation of China (31601089), the Science and Technology Planning Project of Guangdong Province (No. 2015B010109004), the Fundamental Research Funds for the Central Universities (No. 2015ZM177), the Open Project Program of Guangdong Key Laboratory of Fermentation and Enzyme Engineering, SCUT (FJ2015009).

\section{REFERENCES}

1. Ma J, Ma Z, Wang J, Milne RW, Xu D, Davey AK, Evans AM. Isosteviol reduces plasma glucose levels in the intravenous glucose tolerance test in Zucker diabetic fatty rats. Diabetes Obes Metab. 2007; 9:597-99.

2. Wong KL, Yang HY, Chan P, Cheng TH, Liu JC, Hsu FL, Liu IM, Cheng YW, Cheng JT. Isosteviol as a potassium channel opener to lower intracellular calcium concentrations in cultured aortic smooth muscle cells. Planta Medica. 2004; 70:108-12. 
3. Chang SF, Chou BH, Yang LM, Hsu FL, Lin WK, Ho Y, Lin SJ. Microbial transformation of isosteviol oxime and the inhibitory effects on NF-kappaB and AP-1 activation in LPS-stimulated macrophages. Bioorg Med Chem. 2009; 17:6348-53.

4. Zhu SL, Wu Y, Liu CJ, Wei CY, Tao JC, Liu HM. Synthesis and in vitro cytotoxic activity evaluation of novel heterocycle bridged carbothioamide type isosteviol derivatives as antitumor agents. Bioorg Med Chem Lett. 2013; 23:1343-46.

5. Xu D, Li Y, Wang J, Davey AK, Zhang S, Evans AM. The cardioprotective effect of isosteviol on rats with heart ischemia-reperfusion injury. Life Sciences. 2007; 80:269-74.

6. Tan W. The use of kauranes compounds in the manufacture of medicament. Google Patents. 2005; WO2005110383A1.

7. Wong KL, Chan P, Yang HY, Hsu FL, Liu IM, Cheng YW, Cheng JT. Isosteviol acts on potassium channels to relax isolated aortic strips of Wistar rat. Life Sci. 2004; 74:2379.

8. Hausenloy DJ, Yellon DM. Myocardial ischemiareperfusion injury: a neglected therapeutic target. J Clin Invest. 2013; 123:92-100.

9. Kim H, Scimia MC, Wilkinson D, Trelles RD, Wood MR, Bowtell D, Dillin A, Mercola M, Ronai ZA. Fine-tuning of Drp1/Fis1 availability by AKAP121/Siah2 regulates mitochondrial adaptation to hypoxia. Mol Cell. 2011; 44:532-44.

10. Zorzano A, Sebastián D, Segalés J, Palacín M. The molecular machinery of mitochondrial fusion and fission: An opportunity for drug discovery? Curr Opin Drug Discov Devel. 2009; 12:597-606.

11. Ježek P, Plecitá-Hlavatá L. Mitochondrial reticulum network dynamics in relation to oxidative stress, redox regulation, and hypoxia. Int J Biochem Cell Biol. 2009; 41:1790-804.

12. Mockridge JW, Marber MS, Heads RJ. Activation of Akt during simulated ischemia/reperfusion in cardiac myocytes. Biochem Biophys Res Commun. 2000; 270:947-52.

13. Bartz RR, Suliman HB, Piantadosi CA. Redox mechanisms of cardiomyocyte mitochondrial protection. Front Physiol. 2015; 6:291.

14. Karbowski M, Youle RJ. Dynamics of mitochondrial morphology in healthy cells and during apoptosis. Cell Death Differ. 2003; 10:870-80.

15. Perrelli MG, Pagliaro P, Penna C. Ischemia/reperfusion injury and cardioprotective mechanisms: Role of mitochondria and reactive oxygen species. World J Cardiol. 2011; 3:186-200.

16. Frank S, Gaume B, Bergmann-Leitner ES, Leitner WW, Robert EG, Catez F, Smith CL, Youle RJ. The role of dynamin-related protein 1, a mediator of mitochondrial fission, in apoptosis. Dev Cell. 2001; 1:515-25.
17. Lee YJ, Jeong SY, Karbowski M, Smith CL, Youle RJ. Roles of the mammalian mitochondrial fission and fusion mediators Fis1, Drp1, and Opa1 in apoptosis. Mol Biol Cell. 2004; 15:5001-11.

18. Ong SB, Subrayan S, Lim SY, Yellon DM, Davidson SM, Hausenloy DJ. Inhibiting mitochondrial fission protects the heart against ischemia/reperfusion injury. Circulation. 2010; 121:2012-22.

19. Smirnova E, Griparic L, Shurland DL, van der Bliek AM. Dynamin-related protein Drp1 is required for mitochondrial division in mammalian cells. Mol Biol Cell. 2001; 12:2245-56.

20. Sharp WW, Fang YH, Han M, Zhang HJ, Hong Z, Banathy A, Morrow E, Ryan JJ, Archer SL. Dynamin-related protein 1 (Drp1)-mediated diastolic dysfunction in myocardial ischemia-reperfusion injury: therapeutic benefits of Drp1 inhibition to reduce mitochondrial fission. FASEB J. 2014; 28:316-26.

21. Sharp WW. Dynamin-related protein 1 as a therapeutic target in cardiac arrest. J Mol Med (Berl). 2015; 93:243-52.

22. Kuznetsov AV, Javadov S, Sickinger S, Frotschnig S, Grimm M. H9c2 and HL-1 cells demonstrate distinct features of energy metabolism, mitochondrial function and sensitivity to hypoxia-reoxygenation. Biochim Biophys Acta. 2015; 1853:276-84.

23. Mammucari C, Rizzuto R. Signaling pathways in mitochondrial dysfunction and aging. Mech Ageing Dev. 2010; 131:536-43.

24. Walters AM, Porter GA Jr, Brookes PS. Mitochondria as a drug target in ischemic heart disease and cardiomyopathy. Circ Res. 2012; 111:1222-36.

25. Halestrap AP, McStay GP, Clarke SJ. The permeability transition pore complex: another view. Biochimie. 2002; 84:153-66.

26. Korge P, Honda HM, Weiss JN. Protection of cardiac mitochondria by diazoxide and protein kinase $\mathrm{C}$ : implications for ischemic preconditioning. Proc Natl Acad Sci USA. 2002; 99:3312-17.

27. McCully JD, Wakiyama H, Cowan DB, Federman M, Parker RA, Levitsky S. Diazoxide amelioration of myocardial injury and mitochondrial damage during cardiac surgery. Ann Thorac Surg. 2002; 74:2138-45.

28. Elmore S. Apoptosis: a review of programmed cell death. Toxicol Pathol. 2007; 35:495-516. 